\title{
High Precision Simulation of Near Earth Satellite Orbits for SAR-Applications
}

\author{
Marc Kalkuhl ${ }^{1}$, Katharina Nöh ${ }^{1}$, Otmar Loffeld ${ }^{2}$, and Wolfgang Wiechert ${ }^{1}$ \\ 1 University of Siegen, FOMAAS, \\ 2 University of Siegen, ZESS \\ Paul-Bonatz-Str. 9-11, D-57068 Siegen, Germany \\ wiechert@simtec.mb.uni-siegen.de \\ http://www.simtec.mb.uni-siegen.de
}

\begin{abstract}
In a high resolution SAR-satellite mission it is very important to predict the flight trajectory of the satellite with a very high precision. Typically a SAR-satellite operates in near earth orbits. At this altitude the orbit is influenced by several physical phenomena (gravity, atmosphere, and air friction). The model to simulate these orbits should represent these influences sufficiently well. A detailed model has been build on the basis of the newest CHAMP gravity model and the MSIS86 atmosphere model. As a result of the complexity and the mathematical characteristics of this model it is necessary to implement a high order ODE-solver. Three different kinds of error are investigated to evaluate the predictive power of the model: numerical error due to the approximation scheme, power series truncation error on the gravity model, and statistical error due to parameter sensitivity. To this end an extensive sensitivity analysis of thousands of model parameters is carried out.
\end{abstract}

\section{Introduction}

Elementary models for satellite orbits or celestial mechanics based on Newtons Law of gravitation are well known text book examples for simulation courses and numerical mathematics [1]. They assume an ideal model of the gravitational field with spherical equipotential surfaces whereas air friction is neglected. In contrast the modeling and simulation of real satellite orbits based on realistic gravitational fields and including air friction is still a problem that is not satisfactorily solved.

The development of high precision orbit models becomes necessary when satellites aviate in low altitudes of less than $1000 \mathrm{~km}$ and additionally a very precise tracking of the satellite flight trajectory is required. An important application domain is the synthetic aperture radar (SAR) technique for remote sensing of the earth surface [2]. If it is driven in the interferometric mode SAR can even produce three dimensional maps with a height resolution of some few meters. However, in order to achieve this high resolution the position of the SAR sensor and receiver must be known with a high precision. The baseline which is a vector and reflects the difference in the sensor positions should be precisely 
known, i.e. depending on the geometry of the configuration with an error smaller than a few millimeters (length) and a few (or even a fraction of an) arcsec (roll angle). An error of 1 millimeter in this difference results in a meter difference in the height resolution of the map [3].

This precision can only be achieved by a combination of direct position measurements with flight trajectory computations. Typically GPS signals are used for navigation. However, it is a well known fact that even the best available GPSsystem (DGPS - Differential GPS) has only a precision of 1-6 meters. But in use with SAR-satellites this system is not the first choice for reasons that can't be discussed in this paper. All other GPS-systems have a precision in a magnitude of 10 meters. Thus, measurements are taken in regular time intervals in order to obtain redundant position information. At the same time the flight trajectory between two measurements is predicted by a mathematical model. The fusion of both sources of information is done by extended Kalman filter algorithms with alternating trajectory prediction and position update steps [3]. Clearly, the better the underlying trajectory model of the Kalman filter is the better will be the quality of the position estimate. The central question of this contribution is how precise a near earth satellite orbit can be predicted with state of the art knowledge about gravitation and air friction.

\section{Coordinate Systems}

Consulting the literature on the different physical influences on the satellite orbit (gravitation, atmosphere, air-friction) it turns out that models from different sources (geodesy, meteorology) are formulated for different reference coordinate systems. Here the coordinate systems given in Table 1 are relevant. Details about these coordinate systems and their precise definition can be found in [3].

Table 1. Used Coordinate Systems. Letters $x$ (point of intersection between $0^{\circ}$ longitude and equator), $z$ (point at the North Pole), $y$ (completion of the Cartesian System) indicate Cartesian Coordinates. Letters $\lambda$ (longitude), $\delta$ (latitude) and $r$ (distance between point of origin and emission point) resp. $h$ (height above the earth surface) signify the coordinates in (modified) Spherical Coordinate Systems.

\begin{tabular}{|l|c|c|l|}
\hline Name & Shortcut & Coordinates & Characteristics \\
\hline Inertial System & IS & $x_{\mathrm{IS}}, y_{\mathrm{IS}}, z_{\mathrm{IS}}$ & $\begin{array}{l}\text { stationary } \\
\text { clockwise Cartesian CS }\end{array}$ \\
\hline $\begin{array}{l}\text { Earth } \\
\text { Coordinate System }\end{array}$ & $\mathrm{ECS}$ & $x_{\mathrm{ECS}}, y_{\mathrm{ECS}}, z_{\mathrm{ECS}}$ & $\begin{array}{l}\text { earth-bound } \\
\text { clockwise Cartesian CS }\end{array}$ \\
\hline $\begin{array}{l}\text { Geocentric } \\
\text { Coordinate System }\end{array}$ & $\mathrm{GCS}$ & $\lambda_{\mathrm{GCS}}, \delta_{\mathrm{GCS}}, r_{\mathrm{GCS}}$ & $\begin{array}{l}\text { earth-bound } \\
\text { modified Spherical CS } \\
\text { related to ECS }\end{array}$ \\
\hline $\begin{array}{l}\text { World } \\
\text { Geodetic System }\end{array}$ & WGS & $\lambda_{\mathrm{WGS}}, \delta_{\mathrm{WGS}}, h_{\mathrm{WGS}}$ & $\begin{array}{l}\text { earth-bound } \\
\text { modified Spherical CS } \\
\text { represents earth-ellipsoid }\end{array}$ \\
\hline
\end{tabular}


Explicit formulas are available to transform each coordinate system into the other. Figure [1] shows the different coordinate transformations. Some of them have well established names in the literature. By applying the chain rule each coordinate system can be transformed into each other. All these coordinate transformations have been implemented in

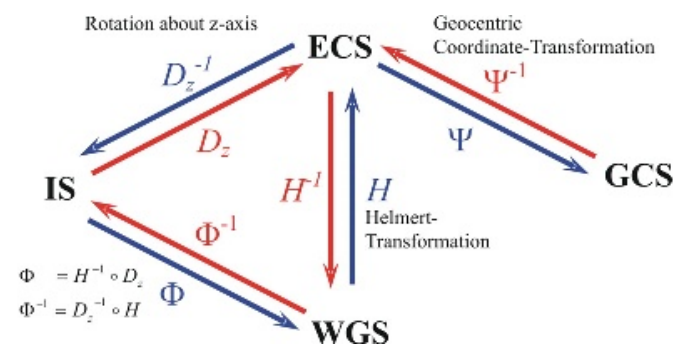

Fig. 1. Transformations between the different used Coordinate Systems.

\section{Gravitation Model}

Several gravitational influences must be taken into account for high precision flight simulation [4]:

- the gravitational field of the earth,

- the direct gravity influence of the moon, sun and planets, and

- the indirect gravity influence of the tide.

For simplicity the following remarks concentrate on the earth gravitational field without any time dependent influences on the flight trajectories.

The earth's gravitational field is described by a potential function $U(\lambda, \delta, r)$ where $\lambda, \delta, r$ are given in GCS coordinates (see Table 1). As an example the classical Newton potential is given by $U=\mu / r$ with $\mu$ - gravitational constant and $r$ - distance between point of origin and emission point.

The most recent and most detailed gravitational model also takes the variation of the gravitational potential in dependency of the longitude and latitude into account. The CHAMP model of the GeoForschungsZentrum Potsdam [5] is based on high precision measurements with the CHAMP-satellite [6]. Here the normalized Legendre functions $\bar{P}_{n, m}$ are used for a series development:

$$
U=\sum_{n=0}^{\infty} \frac{\mu \cdot a_{e}^{n}}{r^{n+1}} \cdot \sum_{m=0}^{n} \bar{P}_{n, m}(\sin (\delta)) \cdot\left(c_{n m} \cdot \cos (m \cdot \lambda)+s_{n m} \cdot \sin (m \cdot \lambda)\right)
$$

with $\mu$ - gravitational constant, $a_{e}$ - earth semi-major axis, $\bar{P}_{n, m}$ - normalized Legendre function and $c_{n m}, s_{n m}$ - harmonic coefficients, $r, \lambda, \delta$ - see Table 1. In general the precision of the gravitation model can be increased by adding further terms to the series development. Currently the parameters are available up to the 120 th degree (e.g. $n=0,1, \ldots, 120$ ) [5]. For example there are 6642 coefficients at level 80 and 14762 for degree 120 . 


\section{Atmosphere Model}

Earth atmosphere is subdivided into several layers from which the thermosphere $(90-500 \mathrm{~km})$ and the exosphere $(>500 \mathrm{~km})$ are important in this context. Satellite orbits are influenced by air friction up to a height of about $1000 \mathrm{~km}$. Remote sensing satellites typically operate in near earth orbit (e.g. ERS in $750 \mathrm{~km}$ altitude (4).

In contrast to gravitation the earth atmosphere is subject to several time dependent influences like the seasons and the sun activity. Frequently the MSIS model 7 is used to calculate the atmospheres density. The MSIS model family is formulated in the WGS coordinate system. The MSIS86 model used here takes the following parameters into account:

$D$ date

UT universal time

$h$ altitude above earth surface

$\delta$ geodetic latitude

$\lambda$ geodetic longitude
$S T L \quad$ local apparent solar time F107A 3 month average of F10.7 flux F107 daily F10.7 flux for prev. day Ap magnetic index

This model can be obtained as a FORTRAN source code from [8]. Its details are much too involved to present them in this paper.

\section{$5 \quad$ Air Friction Model}

In addition to the air density the geometry of the satellite determines the total deceleration due to air friction. In particular the front surface of the satellite depends on its current orientation relative to the flight trajectory. In the used model a constant surface is assumed and the total deceleration is assumed to be proportional to the square of the current velocity which is a common model for very low gas densities close to molecular flow conditions:

$$
\vec{d}=-\frac{1}{2} \cdot c_{D} \cdot \frac{A}{m} \cdot \rho \cdot V_{a} \cdot \vec{V}_{a}
$$

with $m$ - mass of satellite, $\rho$ - air density, $c_{D}$ - drag coefficient, $A$ - aerodynamic active satellite cross sectional area, $V_{a}$ - norm of track speed vector, $\vec{V}_{a}$ - track speed vector relative to atmosphere.

\section{$6 \quad$ Flight Trajectory Model and Implementation}

In total there are three sets of parameters in the present model which influence the flight trajectory:

- degree and coefficients of the gravitational model,

- coefficients of the air density model, and

- satellite geometry coefficients. 
The dynamics of a satellite can now be described by combining gravitational and atmospheric influences into Newtons law of motion:

$$
m \cdot \ddot{\vec{r}}=F_{\text {grav } .}+F_{\text {frict. }}=m \cdot \nabla U+m \cdot \vec{d} .
$$

Notice, that the two forces must be given with respect to the same coordinate system which is chosen here to be the GCS coordinate system. The chain rule must be applied to compute the gradient $\nabla U$ in the GCS system. More details can be found in $[9]$.

The general model (2) has been implemented in MATLAB. To this end the coefficients of the gravitation model are imported from a file obtained directly from the GFZ Potsdam [5]. The MSIS86 model has been converted from the FORTRAN code to a $\mathrm{C}$ code by using the $\mathrm{f} 2 \mathrm{c}$ translator and then coupled to MATLAB via the MEX-interface.

It turned out to be very important to implement the computation of the normalized Legendre function $\bar{P}_{n, m}$ in a numerical stable way. Therefore a recursion formula [9] should be used instead of the explicit formula, which is subject to strong numerical errors and also much slower to compute.

Because the resulting ordinary differential equation system (2) has only 6 dimensions and stiffness is not expected the high order explicit integration algorithm DOPRI8 with step size control and dense output [10] has been implemented in MATLAB. It is currently one of the most frequently used high order Runge-Kutta methods. The average time to simulate one full orbit of a satellite in $800 \mathrm{~km}$ altitude with the CHAMP model of degree 40 was about 30 seconds on an AMD $1200 \mathrm{MHz}$ computer.

\section{Numerical Precision}

Before the influence of the parameter uncertainties on the prediction of the flight trajectories can be evaluated it must be known how precise the numerical solution of the differential equation is. To this end a series of test runs was undertaken.

A good test case can be produced by omitting the friction force from equation (2). A first test was the classical Newton model for which exact solutions are available. If the starting conditions of the system are chosen appropriately the flight trajectory is known to be an ellipse [1. Thus the closing of the trajectory can be checked numerically. The absolute position deviation in the IS system after one full orbit is less than 2 millimeters in each coordinate. Also omitting the friction force in the second test case the system must be conservative with respect to mechanical energy [1. The test investigates the change in the computed energies in relation to the energy at the starting position for simulations with the classical Newton model and CHAMP model of degree 40 for one full orbit. It turned out that for the classical Newton model a numerical precision of $10^{-13}$ is obtained, whereas for the most complex model only a numerical precision of at least four digits is achieved. 




Fig. 2. Smoothness of the right hand side of the differential equation system. Here the longitude component of $\nabla U$ (degree 80 ) for $0^{\circ}$ latitude is shown.
In order to understand the obvious difference between Newton and CHAMP model a closer look is be taken to the smoothness of the right hand side of the differential equation system (21). Clearly, the higher the degree of the CHAMP model is the more high frequent sine and cosine terms are included in (1). This increasingly reduces the smoothness of the model. Figure 2 shows the longitude component of $\nabla U$ for degree 80 in the right hand side of the ODE for $0^{\circ}$ latitude. In contrary to the classical Newton model the real gravitational field is not very smooth. Thus algorithms with an excellent behavior for smooth systems like for example Taylor solvers need not perform well in this case.

\section{Sensitivity Analysis}

Having an estimate of the numerical precision it is now possible to carry out parameter variations in order to judge the model prediction uncertainty.

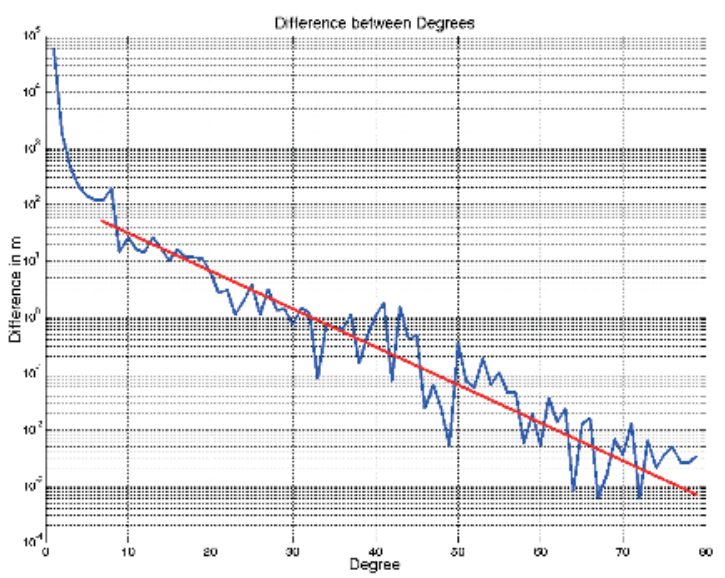

Fig. 3. Absolute deviation in space between two consecutive degrees.
To this end a rigorous sensitivity analysis was carried out for a sample trajectory with realistic starting conditions of a satellite in an altitude of $800 \mathrm{~km}$. The parameters for the atmosphere and air friction model have been set to meaningful average values. Before the parameter sensitivities are computed it is tested how large the series truncation error is, when all parameters are assumed to be exact. Figure 3 shows the absolute deviation in space between two consecutive degrees. A log-linear decrease 
can be extrapolated. As a result of this consideration it can be ascertained that the series truncation error of the gravitation model is in the magnitude of 7 decimeters for degree 40 and 1 millimeter for degree 80.

The quantities for which the sensitivities with respect to a parameter $p$ were computed were the $\mathrm{x}, \mathrm{y}$ and $\mathrm{z}$ position of the satellite after one full orbit around the earth. To obtain a more condensed measure the total deviation in space, that is

$$
S=\sqrt{(\partial x / \partial p)^{2}+(\partial y / \partial p)^{2}+(\partial z / \partial p)^{2}}
$$

was taken in the following figures. Sensitivities are computed from first order differential quotients which gives rise to 6642 different simulation runs for a gravitation model of degree 80 .

Absolute sensitivities in general are not very meaningful in a practical application context because they have different physical units and thus cannot be directly compared to each other. In order to obtain comparable sensitivities each sensitivity is scaled with the precision $\sigma_{p}$ of the respective parameter $p$ :

$$
\bar{S}^{x}=(\partial x / \partial p) \cdot \sigma_{p}, \quad \bar{S}^{y}=(\partial y / \partial p) \cdot \sigma_{p}, \quad \bar{S}^{z}=(\partial z / \partial p) \cdot \sigma_{p}, \quad \bar{S}=S \cdot \sigma_{p} .
$$

Whereas the precision of the gravitational coefficients can be obtained from the GFZ institute, the precision of most other coefficients (air density, satellite geometry) have been roughly estimated. In case of doubt a pessimistic assumption was made (e.g. up to $30 \%$ relative tolerance for some atmosphere parameters). Figure 4 shows the results for the CHAMP model (degree 80) in combination with the atmosphere and air friction sensitivities. In order to condense the information all sensitivities of the parameters in the same polynomial degree were summarized by taking a mean value. Interestingly, the more detailed the gravitational model is the more imprecise the prediction becomes. This effect is based on the fact, that the deviation magnitude $\sigma$ of the gravitation parameters $c_{n m}, s_{n m}$ for degrees greater than 40 becomes equal resp. larger than the parameter himself. However, all these sensitivities are in the same order of magnitude and up

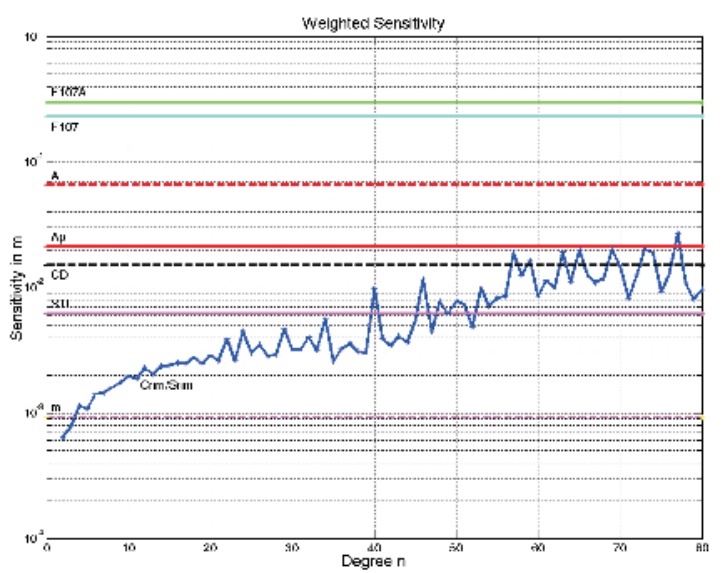

Fig. 4. Computed weighted sensitivities of the CHAMP (degree 80), atmosphere and air friction model parameters after one full orbit. The curve shows the sensitivity of the CHAMP model parameters. The horizontal lines display the different atmosphere (solid) and air friction (dashed) parameter sensitivities. 
to approximately degree 65 are well below the truncation error of the gravitational model. Above this degree the sensitivities exceed the truncation error which means that the model causes more error with its parameters than the series truncation. In comparison the sensitivities of the air friction and satellite geometry parameters are also shown in Figure 4. It turns out that these sensitivities are mostly in the same order of magnitude as the gravitational parameters except for the two parameters $F 107$ and $F 107 A$ which have a significantly higher influence. Unfortunately, these are exactly those parameters where only little knowledge is available today.

\section{Conclusion}

Assuming that the state of the art models for gravitation and air friction are correct representations of reality and the uncertainties in these parameters are well estimated the following conclusions can be drawn. According to the three kinds of error consideration the gravitation model need and should be computed to a maximal degree of 60 for the requirements of this contribution. A higher degree will not yield a higher precision. A consequence out of this is, that the computing time can be reduced compared to a model of higher degrees significantly and numerical errors can be avoided. In addition to that the sensitivity analysis gives also another important result: it's a prime importance to get the atmosphere parameters with a very high precision, because they have a great influence on the whole model.

Future investigation will be made on the consideration of other effects (e.g. moon or sun gravity) in the flight trajectory model. Also the enhancement of the model by reproducing the satellites geometry and inertia is intended.

\section{References}

1. Hairer, E., Lubich, C., Wanner, G.: Geometric Numerical Integration, Springer, 2002

2. Franceschetti, G., Lanari, R., Lanari, R.: Sythetic Aperture Radar Processing, CRC Press, 1999

3. Knedlik, S.: Auf Kalman-Filtern basierende Verfahren zur Erzielung genauerer Höhenmodelle in der SAR-Interferometrie, PhD. Thesis, University of Siegen, 2003

4. Montenbruck, O., Gill, E.: Satellite Orbits, Springer, 2000

5. GeoForschungsZentrum-Potsdam: http://www.gfz-potsdam.de

6. Reigber, Ch., Lühr, H., Schwintzer, P. (eds.): First CHAMP Mission Results for Gravity, Magnetic and Atmospheric Studies. Springer, 120-127, 2003.

7. Hedin, A.E.: MSIS-86 Thermospheric Model, J. Geophys. Res., 1987

8. MSIS86 model description and code download: http://uap-www.nrl.navy.mil/models_web/msis/msis_home.htm

9. Kalkuhl, M.: Erdnahe Orbitsimulation eines Interferometrischen Cart-Wheels, Diploma Thesis, University of Siegen, 2003

10. Hairer, E., Norsett, S.P., Wanner, G.: Solving Ordinary Differential Equations I, 1st edition, Springer, 1993 